\title{
Development of PCR-based markers for discriminating Solanum berthaultii using its complete chloroplast genome sequence
}

\author{
Soojung Kim • Kwang-Soo Cho $\cdot$ Tae-Ho Park
}

Received: 30 June 2018 / Revised: 11 September 2018 / Accepted: 11 September 2018

(c) Korean Society for Plant Biotechnology

\begin{abstract}
Solanum berthaultii is one of the wild diploid Solanum species, which is an excellent resource in potato breeding owing to its resistance to several important pathogens. On the other hand, sexual hybridization between $S$. berthaultii and $S$. tuberosum (potato) is limited because of their sexual incompatibility. Therefore, cell fusion can be used to introgress various novel traits from this wild species into the cultivated potatoes. After cell fusion, it is crucial to identify fusion products with the aid of molecular markers. In this study, the chloroplast genome sequence of $S$. berthaultii obtained by next-generation sequencing technology was described and compared with those of five other Solanum species to develop $S$. berthaultii specific markers. A total sequence length of the chloroplast genome is $155,533 \mathrm{bp}$. The structural organization of the chloroplast genome is similar to those of the five other Solanum species. Phylogenic analysis with 25 other Solanaceae species revealed that $S$. berthaultii is most closely located with $S$. tuberosum. Additional comparison of the chloroplast genome sequence with those of the five Solanum species revealed 25 SNPs specific to $S$. berthaultii. Based on these SNPs, six PCR-based markers for differentiating $S$. berthaultii from other Solanum species were developed. These markers will facilitate the selection of fusion products and accelerate potato breeding using $S$. berthaultii.
\end{abstract}

Keywords PCR-based marker, cpDNA, Potato, SNPs, Solanum berthaultii

* S. Kim and K. $-\mathrm{S}$, Cho contributed equally to this work.

S. Kim • T.-H. Park $(\bowtie)$

Department of Horticulture, Daegu University, Gyeongsan 38453, South Korea

e-mail: thzoo@daegu.ac.kr

K.-S. Cho

Highland Agriculture Research Institute, National Institute of Crop Science, Rural Development Administration, Pyeongchang 25342, South Korea

\section{Introduction}

Potato (Solanum tuberosum L.) is one of the most important crops in the world. Its production has been jeopardized seriously by diverse pathogens, particularly the oomycete pathogen, Phytophthora infestans, the causal agent of late blight (Rauscher et al. 2006). Therefore, potato breeding has focused on achieving high level resistance to late blight. Moreover, several resistant genes have been introgressed to improve the resistance to late blight from wild Solanum species into cultivated potatoes in potato breeding.

S. berthaultii originating from Bolivia, South America is a wild diploid species and a relative of potato (Pavek and Corsini 2001; Spooner and Castillo 1997). The species has been known as one of the important resources for resistance to several important pathogens such as Phytophthora infestans, potato virus $\mathrm{Y}$ etc. in potato breeding (Ewing et al. 2000; Nouri-Ellouz et al. 2016; Park et al. 2009; Rauscher et al. 2010; Tan et al. 2010). On the other hand, sexual incompatibility caused by the different ploidy levels of the genome and endosperm balance number (EBN) between this wild species and potato have restricted the utilization of this wild species for improvements during classical breeding processes (Cho et al. 1997; Oritz and Ehlenfeldt 1992). To overcome this sexual barrier for interspecific gene transfer, therefore, protoplast fusion has been attempted with the two different species for crop improvement (Bidani et al. 2007; Nouri-Ellouz et al. 2016). In potato breeding, this technique has also been applied and succeeded in producing interspecific somatic hybrids such as $S$. tuberosum (+) $S$. chaconese (Chen et al. 2013), S. tuberosum (+) S. brevidens (Barsby et al. 1984), S. tuberosum (+) S. nigrum (Binding et al. 1982), S. tuberosum (+) S. phureja (Putie et al. 1986) and S. tuberosum (+) S. commersonii (Kim-Lee et al. 2005). When somatic hybridization is performed successfully, molecular markers can be used for the identification and verification of the somatic hybrids to confirm the hybridity 
Table 1 Results of comparative analysis of the chloroplast genome sequence of $S$. berthaultii with those of five Solanaceae species

\begin{tabular}{|c|c|c|c|c|c|c|c|}
\hline Species & Accession no. & $\begin{array}{l}\text { Total Length } \\
\text { (bp) }\end{array}$ & $\begin{array}{c}\text { GC content } \\
(\%)\end{array}$ & $\begin{array}{l}\text { Total No. } \\
\text { of genes }\end{array}$ & $\begin{array}{l}\text { No. of } \\
\text { tRNA }\end{array}$ & $\begin{array}{l}\text { No. of } \\
\text { rRNA }\end{array}$ & Reference \\
\hline S. berthaultii & KY419708 & 155,533 & 38 & 137 & 39 & 4 & This study \\
\hline S. nigrum & KM489055 & 155,432 & 38 & 139 & 39 & 4 & Cho and Park (2015) \\
\hline S. commersonii & KM489054 & 155,525 & 37.5 & 133 & 33 & 4 & $\begin{array}{l}\text { Cho et al. } \\
(2016)\end{array}$ \\
\hline S. tubersoum & NC008096 & 155,296 & 37.5 & 131 & 36 & 4 & $\begin{array}{l}\text { Gargano et al. } \\
(2005)\end{array}$ \\
\hline S. tuberosum & KM489056 & 155,312 & 37 & 130 & 30 & 4 & $\begin{array}{l}\text { Cho et al. } \\
\text { (2016) }\end{array}$ \\
\hline S. bulbocastanum & NC007943 & 155,371 & 38 & 133 & 30 & 4 & Daniell et al. (2006) \\
\hline
\end{tabular}

of both nuclear and organelle DNA (Cho and Park 2016; Cho et al. 2016). This process is essential due to the fact that a large amount of nuclear and organelle DNA can be transferred between two sexually incompatible species (Guo et al. 2004).

The chloroplast, a photosynthetic intracellular organelle has a circular double-stranded DNA molecule on its own genome. Most chloroplast genomes contain 110-130 genes encoding up to 80 unique proteins, approximately 30 tRNAs and 4 rRNAs. The angiosperm chloroplast is usually $115 \sim$ $165 \mathrm{~kb}$ in size and has a quadripartite organization comprised of two $12-75 \mathrm{~kb}$ inverted repeats (IR) separating the $80 \sim 90 \mathrm{~kb}$ large single copy (LSC) and 16 27 kb small single copy (SSC) regions (Yurina and Odintosova 1998). As shown in Table 1, several complete chloroplast genome sequences of Solanum species including $S$. tuberosum (KM489056 and NC008096), S. commersonii (KM489055), S. bulbocastanum (NC007943), and S. nigrum (KM489054) have previously been reported (Cho and Park 2016; Cho et al. 2016; Chung et al. 2006; Daniell et al. 2006). Their chloroplast genomes are highly conserved in size, structure and gene organization including the presence of two copies of IRs separated by LSC and SSC regions as previously known (Palmer 1991; Raubeson and Jansen 2005; Saski et al. 2005; Sugiura et al. 1998). Nevertheless, diversification of chloroplast genome sequences derived from the comparison of complete chloroplast genome sequences among plant species provides sufficient information (Cho et al. 2015). In the chloroplast genomes of many plants, a number of mutations such as single nucleotide polymorphism (SNPs), and insertions/deletions (InDels) and structural changes such as inversions, and rearrangements of gene order have been reported (Calsa Junior et al. 2004; Jheng et al. 2012; Kim et al. 2005; Saski et al. 2005).

Previously, the chloroplast genome sequence of $S$. berthaultii was completed using next-generation sequencing technology and shortly announced (Park 2017). In this study, we described the result of the sequence in detail, compared it with those of other Solanaceae species, and developed specific markers for $S$. berthaultii.

\section{Materials and Methods}

Plant Materials and DNA Isolation

Plant materials of 22 genotypes of Solanum species such as S. tuberosum (PT56), S. berthaultii (PI310981; SB1-1, -2, -4 and -6), S. acaule (PI310970; SA-2), S. pinnatisectum (PI190115; SP-12), S. hjertingii (PI186559; SH1-15), S. mochiquense (PI338616; SM1-6), S. cardiophyllum (PI341233; SC1-2), S. verrucosum (PI160228; SV1-4), S. kurtzianum (PI498422; SK-5), S. stoloniferum (PI160224; SS-1), S. hougassi (PI161174; SH2-10), S. microdontum (PI310979; SM2-3), S. commersonii (PI558050; SC2-7), S. chacoense (PI201846; SC3-6), S. demissum (PI218047; SD-9), S. brevicaule (PI205394; SB2-7), S. vernei (PI230468; SV2-4), S. jamesii (PI578236; SJ-1) and $S$. tuberosum subsp. andigenum (PI566805; ST2-1) provided by the Highland Agriculture Research Institute, South Korea were used to compare molecular markers. They were cultivated in a greenhouse and cultured in vitro. For DNA isolation, fresh leaves collected from the greenhouse or in vitro plants were directly used or freeze-dried, ground and stored at $-80^{\circ} \mathrm{C}$ when they were needed. Approximately $100 \mathrm{mg}$ of fresh leaves were applied for the extraction of the total genomic DNA using a Genomic DNA Extraction kit for plants (RBC, New Taipei City, Taiwan).

Chloroplast Genome Sequencing

Sequencing was conducted using the Illumina Hiseq2000 
(Illumina, SanDiego, CA, USA) platform at Macrogen (Macrogen, Seoul, South Korea) with the total genomic DNA of $S$. berthaultii (SB1-1). The chloroplast genome sequence was obtained by de novo assembly via the Phyzen bioinformatics pipeline (http://phyzen.com). A CLC assembler (ver. 4.06 beta, CLC Inc, Rarhus, Denmark) was used for de novo assembly. From the overall reads, reads with raw scores of 20 or less were discarded using the CLC-quality trim tool and the parameters of a minimum (300 1,000 bp) autonomously controlled overlap size were applied. From the total contigs, the principal contigs representing the chloroplast genome were retrieved by Nucmer (Kurtz et al. 2004) using the chloroplast genome sequence of $S$. tuberosum (KM489056) as the reference sequence (Cho et al. 2016). The results from BLASTZ analysis with the reference sequence arranged the representative chloroplast contigs in order (Schwartz et al. 2003) and they were connected to a single draft sequence by joining the overlapping terminal sequences followed by manual editing through a comparison with the chloroplast genome sequence of $S$. tuberosum (KM489056) reported by Cho et al. (2016). Gene annotation was conducted using DOGMA (Wyman et al. 2004) and the circular map of the $S$. berthaultii chloroplast genome was generated using the software OrganellarGenomeDRAW (http://ogdraw.mpimp-golm.mpg.de) (Lohse et al. 2013).

Comparison of Chloroplast Genome Sequences

The chloroplast genome sequence of $S$. berthaultii identified in this study was compared with those of 25 Solanaceae species including eight Solanum species, S. tuberosum (DQ386163), S. commersonii (KM489054), S. bulbocastamum (NC007943), S. lycopersicum (DQ347959), S. pimpinellifolium (NC026882), S. peruvianum (KP117026), S. nigrum (KM 489055), and $S$. melongena (KU682719) obtained from the National Center for Biotechnology Information (NCBI). Sequence comparison and phylogenetic analysis were conducted using the chloroplast coding sequences of eight species by MEGA 6.0 (Tamura et al. 2013) and by a maximum likelihood and a maximum parsimony methods under PAUP* v4.0b10 (Swofford 2001), respectively. A total of 1,000 bootstap replicates and a $50 \%$ cutoff value for the bootstrap consensus tree were applied for the analysis. Multiple alignment was also performed using the complete chloroplast genome sequences of six species by ClustalW2 in EMBL (https://www.ebi.ac.uk/Tools/msa/clustalw2).

\section{Development of PCR-Based Markers}

The S. berthaultii specific SNPs were identified with the results of multiple alignment with five Solanum species: $S$. berthaultii (KY419708), S. tuberosum (KM489056 and NC 008096), S. commersonii (KM489054), S. bulbocastanum (DQ347958), and S. nigrum (KM489055). After checking whether or not the proper restriction enzymes, which could digest only a SNP region on the sequence of $S$. berthaultii or on the sequence of other four Solanum species, existed using dCAPS Finder 2.0 (http://helix.wustl.edu/dcaps/dcaps. $\mathrm{html})$, the primers covering each SNP region were designed. PCR was routinely performed with the 22 genotypes (PT56, SB1-1, SB1-2, SB1-4, SB1-6, SA-2, SP-12, SH1-15, SM1-6, SC1-2, SV1-4, SK-5, SS-1, SH2-10, SM2-3, SC2-7, SC3-6, SD-9, SB2-7, SV2-4, SJ-1, and ST2-1) as described by Cho and Park (2016). The DNA fragments in the PCR products were separated on $1 \%$ agarose gel and detected using the nucleic acid staining solution RedSafe (Intron Biotechnology, Seongnam, South Korea). When all 22 genotypes were amplified with certain primers, the selected restriction enzymes were applied to the PCR products. The digested fragments were separated by electrophoresis on $1 \%$ agarose gel and visualized under UV lights.

\section{Results and Discussion}

Complete Chloroplast Genome Sequence of S. berthaultii

The complete chloroplast genome sequencing of $S$. berthaultii was performed using NGS (next generation sequencing) technology. A library produced by the Illumina PE standard protocol generated 2,506,011,690 bp sequences in total and their average read length was $348 \mathrm{bp}$. Three representative contigs were identified using the de novo assembly of paired end sequences of the entire genome. They covered the entire chloroplast genome sequence of $S$. tuberosum (KM489056) reported by Cho et al. (2016) and arranged in order based on the results of BLASTZ analysis (Schwartz et al. 2003) (Fig. 1). The putative assembly errors curated by mapping 1,699.85x raw reads onto the final assembly, and PCR products of several regions including border sequences of a pair of inverted repeats (IRs), a small single copy region (SSC) and a large single copy region (LSC) validated by BigDye Terminator Cycle Sequencing with ABI3730 automated capillary sequencer finalized the completion of the chloroplast genome sequence. As a part of the results has shortly been announced (Park 2017), the size of the complete chloroplast genome sequence of $S$. berthaultii (GenBank accession no. KY419708) is 155,533 bp. It has the typical quadripartite structure including 25,593 bp IRs separated by SSC of 18,372 bp and LSC of 85,975 bp with a circular 


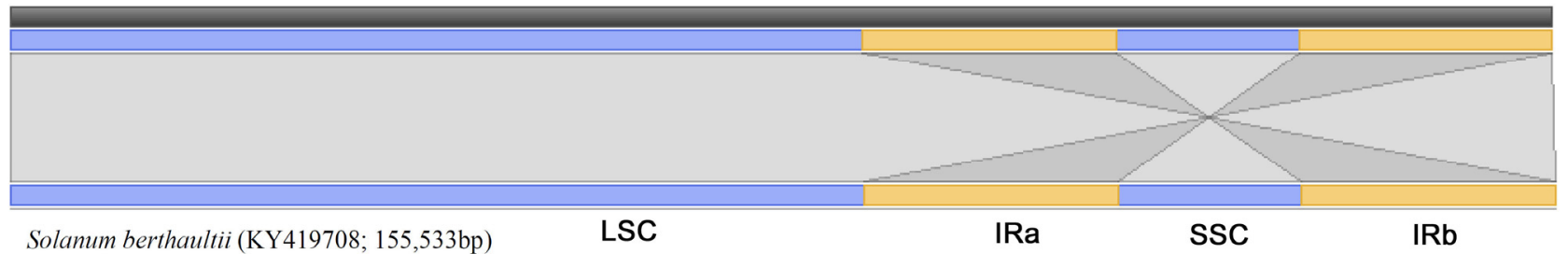

Fig. 1 Assembly result of the complete chloroplast genome sequence of $S$. berthaultii. The four representative contigs (LSC, IRa, $\mathrm{SSC}$, and IRb) for the chloroplast genome of $S$. berthaultii and comparison with the corresponding regions of the $S$. tuberosum chloroplast genome (KM489056) were combined. The blue and yellow bars indicate the contig matching with the reference sequence in the forward and reverse orientation, respectively and the corresponding positions are present at the sequence of $S$. berthaultii

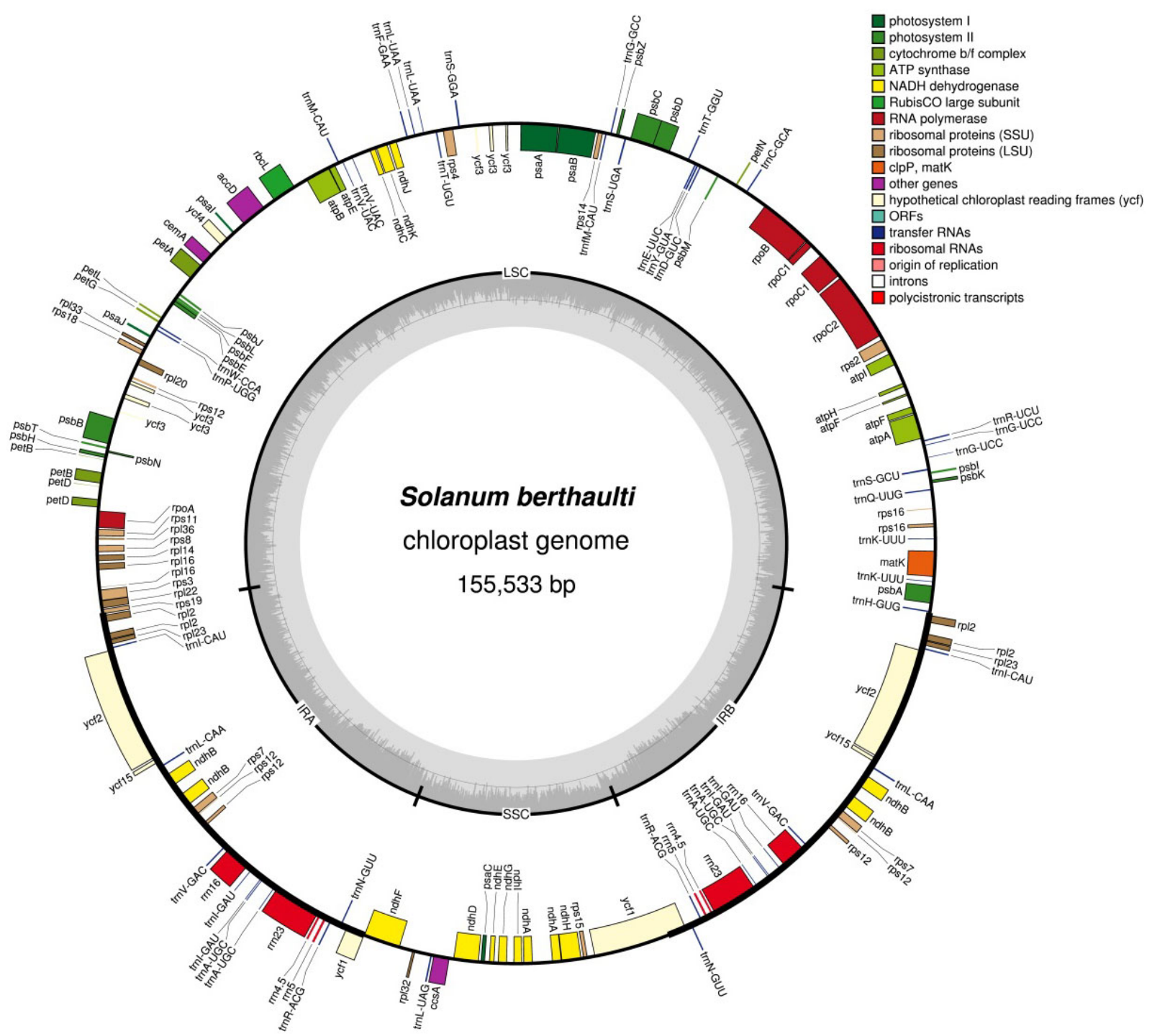

Fig. 2 Gene map of the $S$. berthaultii chloroplast genome. The genes on the outside of the map are transcribed in the clockwise direction and the genes on the inside of the map are transcribed in the counterclockwise direction

double stranded DNA molecule as usual in most plant plastids. The total length is slightly longer than those of other Solanum species (Table 1). The chloroplast genome of $S$. berthaultii contains 137 unique genes and 11 of them are duplicated in the IRs (Fig. 2). Fourty-eight tRNAs and eight rRNAs exist and nine and four of them are duplicated and inversely oriented in the IRs (Fig. 2 and Table 1). $59.2 \%$ coding region of the genome was identified and an 


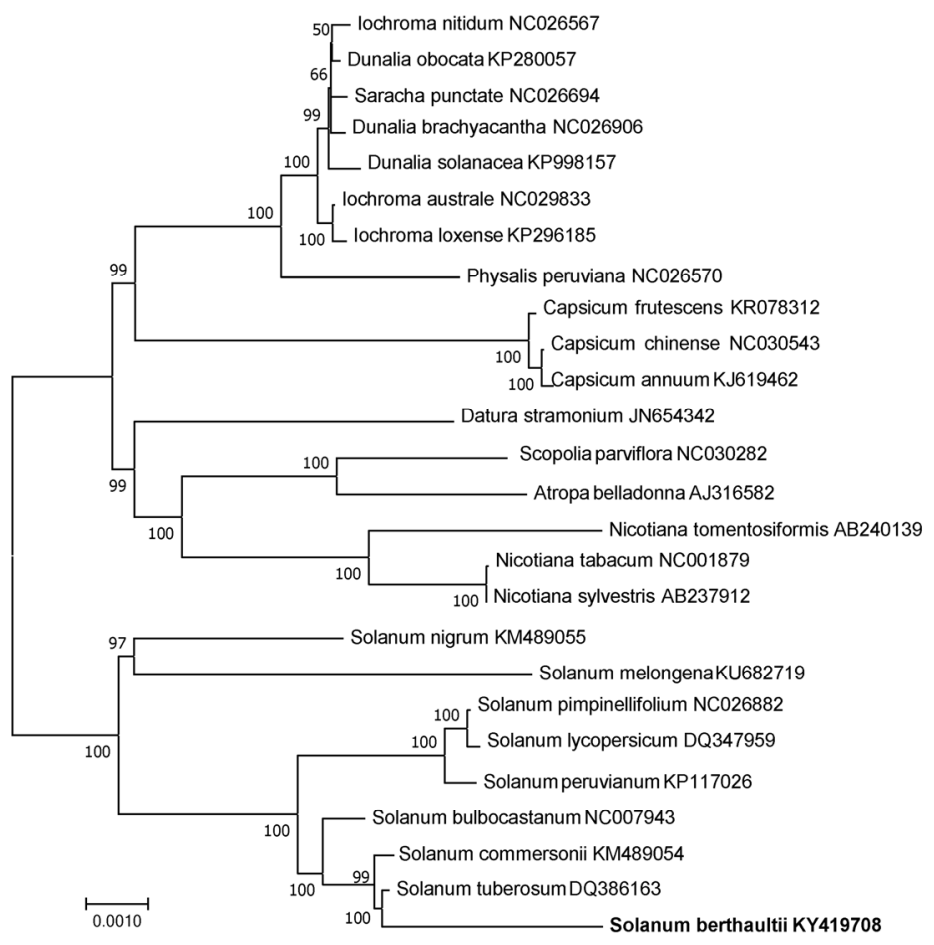

Fig. 3 Maximum-likelihood phylogenetic tree of $S$. berthaultii and 25 species belonging to the Solanaceae family based on protein-coding sequences of each chloroplast genome. The numbers on each node indicate the bootstrap values from 1,000 replicates

average size of the coding sequences is $583 \mathrm{bp}$. It consists of $51.6 \%$ protein coding genes with an average size of 508 bp and $7.7 \%$ RNA genes with an average size of 214 bp. The GC content of the genome is $37.88 \%$ (Table 1 ). As reported by Cho et al. (2016) and Cho and Park (2016), the total length, the GC content, and the total number of predicted genes, tRNA and rRNA of the $S$. berthaultii chloroplast genome are highly conserved with those of other Solanum species.

Phylogenetic and Comparative Analysis of the Chloroplast Genome Sequences

As shown in Figure 3, the phylogenetic relationship was analyzed using chloroplast coding sequence of $S$. berthaultii and those of the 25 Solanaceae species. The maximum parsimony and maximum likelihood analyses produced the same topology and most nodes of the phylogenetic tree were strongly supported by the high bootstrap values. The results showed that $S$. berthaultii belonged to the same clade in Solanum species as expected and $S$. berthaultii formed the sister of $S$. tuberosum. Interestingly, $S$. berthaultii was the closest species to $S$. tuberosum even though it was S. commersonii (Cho et al. 2016).

The complete sequence of the $S$. berthaultii chloroplast genome was compared with those of the five other Solanum species including two different genotypes of $S$. tuberosum to develop molecular markers for the selection of $S$. berthaultii cytoplasm as a reasonable number of InDels or SNPs was previously detected by the complete alignment of the chloroplast genome sequences including both the coding and non-coding regions of the Solanum species (Chung et al., 2006; Cho and Park, 2016). A result of multiple alignment with six species generated by ClustalW2 in EMBL (https:// www.ebi.ac.uk/Tools/msa/clustalw2) showed that overall 39 and 1,788 regions for InDels and SNPs existed among the six Solanum species, respectively.

Discovery and Verification of Specific Molecular Markers for Distinguishing $S$. berthaultii from Potato and Other Wild Solanum Species

The multiple alignment of the chloroplast genome sequences of six different Solanum species revealed a lot of InDels and SNPs among the species. On the other hand, the InDels and SNPs were caused mostly by the sequences of $S$. nigrum or S. bulbocastanum (detail data not shown). This result was supported by the fact that those two species were farther phylogenetically from $S$. berthaultii than $S$. commersonii and $S$. tuberosum as shown in the result of phylogenetic tree (Fig. 3). Therefore, the criteria to search for the InDels or SNPs in which the sequences should be specific to $S$. 
Table 2 Information of the primers and restriction enzymes to generate S. berthaultii specific markers

\begin{tabular}{|c|c|c|c|c|c|}
\hline Marker name & Region & Strand & Primer sequence & $\begin{array}{l}\text { Size } \\
(\mathrm{bp})^{\mathrm{a}}\end{array}$ & $\mathrm{RE}^{\mathrm{b}}$ \\
\hline \multirow{2}{*}{ Sber_CAPS1 } & \multirow{2}{*}{$\begin{array}{l}\text { rps16-trnQ } \\
\text { (Intergenic) }\end{array}$} & Forward & GATTCTGCATCTTCACGTG & \multirow{2}{*}{488} & \multirow{2}{*}{ Cla } \\
\hline & & Reverse & TCTTAATCAATGAGGTAGGC & & \\
\hline \multirow{2}{*}{ Sber_CAPS3 } & \multirow{2}{*}{$\begin{array}{c}\text { rpoCl } \\
\text { (Intragenic) }\end{array}$} & Forward & TGGAACCATGACATATTTGC & \multirow{2}{*}{573} & \multirow{2}{*}{ Apol } \\
\hline & & Reverse & TGTGAAAGAATTTTTGGTCC & & \\
\hline \multirow{2}{*}{ Sber_CAPS5 } & \multirow{2}{*}{$\begin{array}{c}a c c D-p s a I \\
\text { (Intergenic) }\end{array}$} & Forward & AATAAAACAGTACCCGAAGG & \multirow{2}{*}{590} & \multirow{2}{*}{ MseI } \\
\hline & & Reverse & TGTCTTTTGTTCTTGTCTTC & & \\
\hline \multirow{2}{*}{ Sber_CAPS6 } & \multirow{2}{*}{$\begin{array}{c}\text { accD-psaI } \\
\text { (Intergenic) }\end{array}$} & Forward & TTGGGTCGGATTCGAACG & \multirow{2}{*}{570} & \multirow{2}{*}{$X m n \mathbf{I}$} \\
\hline & & Reverse & GATACAAGTCCACGTTTTC & & \\
\hline \multirow{2}{*}{ Sber_CAPS7 } & \multirow{2}{*}{$\begin{array}{c}y c f 4 \\
\text { (Intragenic) }\end{array}$} & Forward & TGGATGGCTGAAATGTAGTC & \multirow{2}{*}{577} & \multirow{2}{*}{ TaiI } \\
\hline & & Reverse & ATCCCCAACGAAAAATACAG & & \\
\hline \multirow{2}{*}{ Sber_CAPS14 } & \multirow{2}{*}{$\begin{array}{c}y c f 1 \\
\text { (Intragenic) }\end{array}$} & Forward & ATAAATGAATTTCСССТTTC & \multirow{2}{*}{373} & \multirow{2}{*}{$M n l$} \\
\hline & & Reverse & ATCTGAAAGCAAACAAATGC & & \\
\hline
\end{tabular}

${ }^{\text {a }}$ Size (bp) indicates the expected sizes of PCR fragments determined based on the $S$. berthaultii sequence.

${ }^{b} \mathrm{RE}$ indicates restriction enzymes generating $S$. berthaultii specific markers by cutting the $S$. berthaultii specific SNPs.

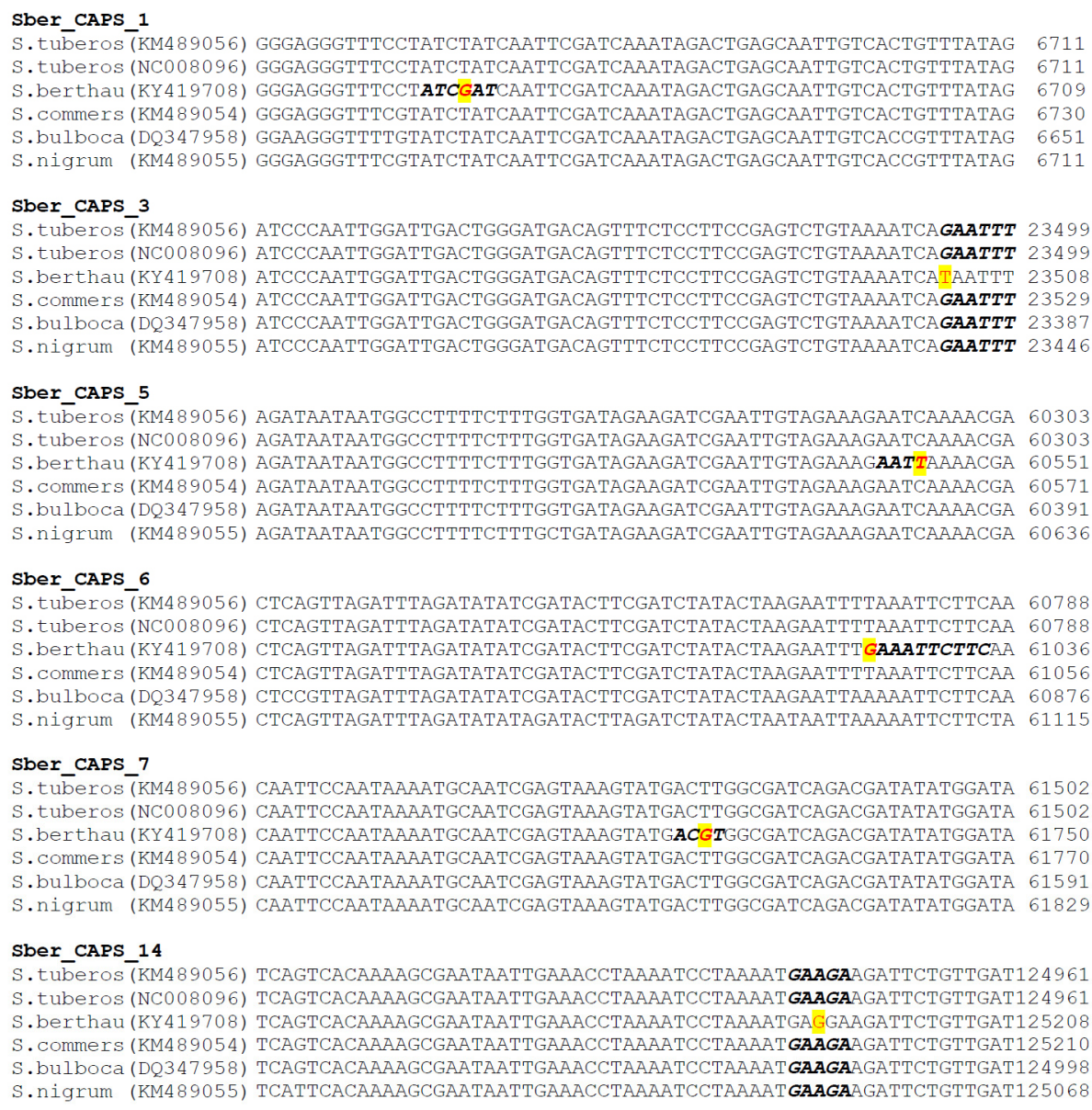

S.tuberos (KM489056) CAATTCCAATAAAATGCAATCGAGTAAAGTATGACTTGGCGATCAGACGATATATGGATA 61502 s.tuberos (NC008096) CAATTCCAATAAAATGCAATCGAGTAAAGTATGACTTGGCGATCAGACGATATATGGATA 61502 S.berthau (KY419708) CAATTCCAATAAAATGCAATCGAGTAAAGTATGACGTGGCGATCAGACGATATATGGATA 61750 S. commers (KM489054) CAATTCCAATAAAATGCAATCGAGTAAAGTATGACTTGGCGATCAGACGATATATGGATA 61770 S.bulboca (DQ347958) CAATTCCAATAAAATGCAATCGAGTAAAGTATGACTTGGCGATCAGACGATATATGGATA 61591 S.nigrum (KM489055) CAATTCCAATAAAATGCAATCGAGTAAAGTATGACTTGGCGATCAGACGATATATGGATA 61829

Fig. 4 Multiple alignment of the sequences on the intergenic and intragenic regions containing SNPs used to develop the CAPS markers. The chloroplast genome sequences of S. tuberosum (KM489056 and NC008096), S. berthaultii (KY419708), S. commersonii (KM489054), S. bulbocastanum (DQ347958), and S. nigrum (KM489055) were used and listed from top to bottom in each region of the CAPS markers. The regions of the SNPs detected on that of $S$. berthaultii are highlighted and the restriction sites are indicated in italic and bold 


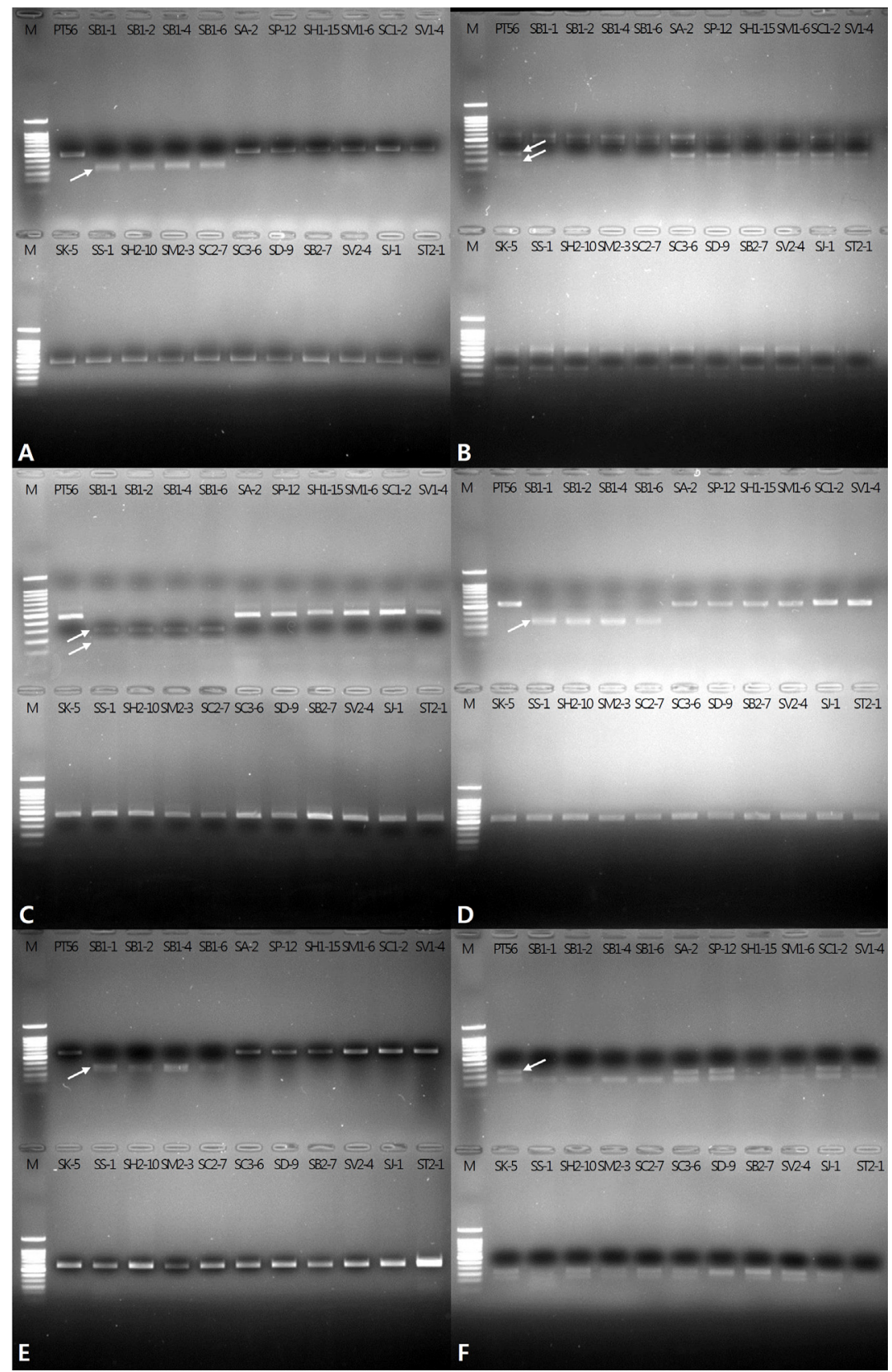

Fig. 5 PCR-based markers for the discrimination of S. berthaultii from other Solanum species. A: Sber_CAPS1. B: Sber_CAPS3. C: Sber_CAPS5. D: Sber_CAPS6. E: Sber_CAPS7. F: Sber_CAPS14. Sber_CAPS1, Sber_CAPS5, Sber_CAPS6, and E. Sber_CAPS7 are positively specific to $\bar{S}$. berthaultii and Sber_CAPS3 and Sber_CAPS14 are negatively specific to $\bar{S}$. berthaultii. The names of the markers correspond to those listed in Table 2 . M, PT56, SB1(-1, -2, -4 and -6), SA-2, SP-12, SH1-15, SM1-6, SC1-2, SV1-4, SK-5, SS-1, SH2-10, SM2-3, SC2-7, SC3-6, SD-9, SB2-7, SV2-4, SJ-1 and ST2-1 indicate a 100bp marker ladder (Bioneer, Daejeon, South Korea), a breeding line of $S$. tuberosum, four different lines of S. berthaultii (PI310981), S. acaule (PI310970), S. pinnatisectum (PI190115), S. hjertingii (PI186559), S. mochiquense (PI338616), S. cardiophyllum (PI341233), S. verrucosum (PI160228), S. kurtzianum (PI498422), S. stoloniferum (PI160224), S. hougassi (PI161174), S. microdontum (PI310979), S. commersonii (PI558050), S. chacoense (PI201846), S. demissum (PI218047), S. brevicaule (PI205394), S. vernei (PI230468), S. jamesii (PI578236), and S. tuberosum subsp. andigenum (PI566805), respectively

berthaultii, but mostly conserved in the five other Solanum species.

Only two of the 39 InDels detected were specific to $S$. berthaultii, but PCR-based molecular markers could not be developed using InDel specific primers. One base pair in the sequence of $S$. berthaultii was different from those of $S$. tuberosum and $S$. commersonii, 19 base pairs from that of $S$. bulbocastanum and six base pairs from that of $S$. nigrum on the first InDel region. Two base pairs in the sequence of $S$. berthaultii were different from those of $S$. 
tuberosum and $S$. commersonii, five base pairs from that of $S$. bulbocastanum and 438 base pairs from that of $S$. nigrum on the second InDel region. Therefore, this study focused on the SNPs identified among six species to develop PCR-based markers. A total of 25 of the 1,788 SNPs detected were specific to $S$. berthaultii. Five SNPs including four consecutive base pairs existed on the inverted repeat region. The regions on which the SNPs existed were distributed almost equally in both coding regions and noncoding regions throughout the entire chloroplast genome sequence although the number of InDels in the non-coding regions was reported to be detected much more than that in the coding regions of the chloroplast genome of Solanum species (Cho and Park 2016; Chung et al. 2006).

The application of PCR to SNPs can be used effectively to develop molecular markers after the PCR products are digested with the appropriate restriction enzymes on the site of the SNPs revealing polymorphisms, which is called the CAPS (cleaved amplified polymorphism sequence) marker (Konieczny and Ausubel, 1993). The CAPS is applied broadly to develop polymorphic markers that can be used to compare different genotypes on Solanum species (Park et al. 2005; Smilde et al. 2005; Uribe et al. 2014) and other plant species (Komori and Nitta 2005; Uncu et al. 2015; Wang et al. 2017). Therefore, 11 primers pairs covering each SNPs site onto which the proper restriction enzymes had been identified were designed. The restriction enzymes could digest only $S$. berthaultii or other Solanum species except $S$. berthaultii. The primers were used for PCR with four different lines of $S$. berthaultii (SB1-1, -2, -3, and -4), S. tuberosum (PT56), $S$. acaule (SA-2), $S$. pinnatisectum (SP-12), S. hjertingii (SH1-15), S. mochiquense (SM1-6), S. cardiophyllum (SC1-2), S. verrucosum (SV1-4), S. kurtzianum (SK-5), S. tuberosum line, S. stoloniferum (SS-1), S. hougassi (SH2-10), S. microdontum (SM2-3), S. commersonii (SC2-7), S. chacoense (SC3-6), S. demissum (SD-9), S. brevicaule (SB2-7), S. vernei (SV2-4), S. jamesii (SJ-1), and S. tuberosum subsp. andigenum (ST2-1) and the restriction enzymes for each SNPs were applied to the PCR products. Finally, six CAPS markers were identified (Table 2 and Fig. 5). Four primer combinations and restriction enzymes that produced distinct bands in the four $S$. berthaultii lines, but not in the other Solanum species including $S$. tuberosum (PT56) were selected (Table 2, Fig. 5A, 5C, 5D and 5E) and two others were vice versa (Table 2, Fig. 5B and 5F).

Characterization of the chlorotype using the molecular markers is important for not only potato breeding, but also evolutionary studies in Solanum species (Bohs and Olmstead 1997; Hosaka and Sanetomo 2012). In previous studies, a random distribution of the chloroplast genome from a certain genotype during in vitro plant regeneration and somatic fusion was commonly observed in plant species including Solanum species, even though a high frequency of recombination occurred in the mitochondrial genome (Chen et al. 2013; Cho et al. 2016; Lössl et al. 2000; Mohapatra et al. 1998; Smyda-Dajmund et al. 2016; Xiang et al. 2004). Overall, the results suggest that the SNP-derived CAPS markers developed in this study can be used to discriminate $S$. berthaultii reliably from other Solanum species, to select the proper chlorotype from the fusion products between $S$. berthaultii and Solanum species and to facilitate potato breeding using $S$. berthaultii.

\section{Acknowledgments}

This study was supported by Korea Institute of Planning and Evaluation for Technology in Food, Agriculture, Forestry and Fisheries (IPET) through Golden Seed Project funded by Ministry of Agriculture, Food and Rural Affairs (MAFRA), Ministry of Oceans and Fisheries (MOF), Rural Development Administration (RDA) and Korea Forest Service (KFS), South Korea (Project No. 213009-05-2-WT411).

\section{References}

Barsby TL, Shepard JF, Kemble RJ, Wong R (1984) Somatic hybridization in the genus Solanum: $S$. tuberosum and $S$. brevidens. Plant Cell Rep 3:165-167

Bidani A, Nouri-Ellouz O, Lakhoua L, Shihachakr D, Cheniclet C, Mahjoub A, Drira N, Gargouri-Bouzid R (2007) Interspecific potato somatic hybrids between Solanum berthaultii and Solanum tubersoum L. showed recombinant plastome and improved tolerance to salinity. Plant Cell Tiss Organ Cult 91:179-189

Binding H, Jain SM, Finger J, Mordhorst G, Nehls R, Gressel J (1982) Somatic hybridization of an atrazine resistant biotype of Solanum nigrum with Solanum tuberosum. Theor Appl Genet 63:273-277

Bohs L, Olmstead RG (1997) Phylogenetic relationships in Solanum (Solanaceae) based on $n d h F$ sequences. Syst Bot 22:5-17

Calsa Junior T, Carraro DM, Benatti MR, Barbosa AC, Kitajima JP, Carrer H (2004) Structural features and transcript-editing analysis of sugarcane (Saccharum officinarum L.) chloroplast genome. Curr Genet 46:366-373

Chen L, Guo X, Xie C, He L, Cai X, Tian L, Song B, Liu J (2013) Nuclear and cytoplasmic genome components of Solanum tuberosum + S. chacoense somatic hybrids and three SSR alleles related to bacterial wilt resistance. Theor Appl Genet 


\section{6:1861-1872}

Cho KS, Cheon KS, Hong SY, Cho JH, Im JS, Mekapogu M, Yu YS, Park TH (2016) Complete chloroplast genome sequences of Solanum commersonii and its application to chloroplast genotype in somatic hybrids with Solanum tuberosum. Plant Cell Rep 35:2113-2123

Cho HM, Kim-Lee HY, Om YH, Kim JK (1997) Influence of endosperm balance number $(\mathrm{EBN})$ in interploidal and interspecific crosses between Solanum tuberosum dihaploids and wild species. Korean J Breed 29:154-161

Cho KS, Park TH (2016) Complete chloroplast genome sequence of Solanum nigrum and Development of markers for the discrimination of $S$. nigrum. Hort Environ Biotechnol 57:69-78

Cho K-S, Yun B-K, Yoon Y-H, Hong S-Y, Mekapogu M, Kim $\mathrm{K}-\mathrm{H}$, Yang T-J (2015) Complete chloroplast genome sequence of tartary Buckwheat (Fagopyrum tataricum) and comparative analysis with common Buckwheat (F. esculentum). PloS One 10:e0125332

Chung HJ, Jung JD, Park HW, Kim JH, Cha HW, Min SR, Jeong WJ, Liu JR (2006) The complete chloroplast genome sequences of Solanum tuberosum and comparative analysis with Solanaceae species identified the presence of a 241-bp in cultivated potato chloroplast DNA sequence. Plant Cell Rep 25:1369-1379

Daniell H, Lee S-B, Grevich J, Saski C, Quesada-Vargas T, Guda C, Tomkins J, Jansen PK (2006) Complete chloroplast genome sequences of Solanum bulbocastanum, Solanum lycopersicum and comparative analyses with other Solanaceae genomes. Theor Appl Genet 112:1503-1518

Ewing EE, Simko I, Samrt CD, Bonierbale MW, Mizubuti ESG, May GD, Fry WE (2000) Genetic mapping from field tests of qualitative and quantitative resistance to Phytophthora infestans in a population derived from Solanum tuberosum and Solanum berthaultii. Mol Breeding 6:25-36

Guo WW, Cai XD, Grosser JW (2004) Somatic cell cybrids and hybrids in plant improvement, p. 635-659. In: H. Daniell, C. Chase (eds.) Molecular biology and biotechnology of plant organelles. Springer, Dordrecht, The Netherlands.

Hosaka K, Sanetomo R (2012) Development of a rapid identification method for potato cytoplasm and its use for evaluating Japanese collections. Theor Appl Genet 125:1237-1251

Jheng C-F, Chen T-C, Lin J-Y, Chen T-C, Wu W-L, Chang C-C (2012) The comparative chloroplast genomic analysis of photosynthetic orchids and developing DNA markers to distinguish Phalaenopsis orchids. Plant Sci 190:62-73

Kim KJ, Choi KS, Jansen RK (2005) Two chloroplast DNA inversion originated simultaneously during the early evolution of the sunflower family (Asteraceae). Mol Biol Evol 22: 1783-1792

Kim-Lee H, Moon JS, Hong YJ, Kim MS, Cho HM (2005) Bacterial wilt resistance in the progenies of the fusion hybrids between haploid of potato and Solanum commersonii. Amer J Potato Res 82:129-137

Komori T, Nitta N (2005) Utilization of the CAPS/dCAPS method to convert rice SNPs into PCR-based markers. Breed Sci 55:93-98

Konieczny A, Ausubel FM (1993) A procedure for mapping Arabidopsis mutations using co-dominant ecotype-specific PCR-based markers. Plant J 4:403-410

Kurtz S, Phillippy A, Delcher AL, Smoot M, Shumway M, Antonescu C, Salzberg SL (2004) Versatile and open software for comparing large genomes. Genome Biol 5:R12

Lohse M, Drechsel O, Kahlau S, Bock R (2013) Organellar GenomeDRAW - a suite of tools for generating physical maps of plastid and mitochondrial genomes and visualizing expression data sets. Nucleic Acids Res 41:W575-W581

Lössl A, Götz A, Braun A, Wenzel G (2000) Molecular markers for cytoplasm in potato: male sterility and contribution of different plastid-mitochondrial configurations to starch production. Euphytica 116:221-230

Mohapatra T, Kirti PB, Dinesh Kumar V, Prakash S, Chopra VL (1998) Random chloroplast segregation and mitochondrial genome recombination in somatic hybrid plants of Diplotaxis catholica + Brassica juncea. Plant Cell Rep 17:814-818

Nouri-Ellouz O, Triki MA, Jbir-Koubaa R, Louhichi A, Charfeddine S, Drira N, Gargouri-Bouzid R (2016) Somatic hybrids between potato and $S$. berthaultii show partial resistance to soil-borne fungi and potato virus Y. J Phythpathol 164:485-496

Ortiz R, Ehlenfeldt MK (1992) The importance of endorsperm balance number in potato breeding and the evolution of tuber-bearing Solanum species. Euphytica 60:105-113

Palmer JD (1991) Plastid chromosomes: structure and evolution, $\mathrm{p}$. 5-53. In: L. Bogorad, K. Vasil (eds.) The molecular biology of plastids. Academic Press, San Diego, USA.

Park T-H (2017) The complete chloroplast genome of Solanum berthaultii, one of the potato wild relative species. Mitochondr DNA Part B 2:88-89

Park T-H, Foster S, Brigneti G, Jones JDG (2009) Two distinct potato late blight resistance genes from Solanum berthaultii are located on chromosome 10. Euphytica 165:269-278

Park T-H, Gros J, Sikkema A, Vleeshouwers VGAA, Muskens M, Allefs S, Jacobsen E, Visser RGF, van der Vossen EAG (2005) The late blight resistance locus Rpi-blb3 from Solanum bulbocastanum belongs to a major late blight $R$ gene cluster on chromosome 4 of potato. Mol Plant-Microb Interact 18:722-729

Pavek JJ, Corsini DL (2001) Utilization of potato genetic resources in variety development. Amer J Potato Res 78:433-441

Puite KJ, Roest S, Pijnacker LP (1986) Somatic hybrid potato plants after electrofusion of diploid Solanum tuberosum and Solanum phureja. Plant Cell Rep 5:262-265

Raubeson LA, Jansen RK (2005) Chloroplast genomes of plants, p. 45-68. In: H. Henry (ed.) Diversity and evolution of plants: genotypic and phenotypic variation in higher plants. CABI Publishing, Wallingford, UK.

Rauscher GM, Samrt CD, Simko I, Bonierbale M, Mayton H, Greenland A and Fry WE (2006) Characterization and mapping of $R_{p i-b e r}$, a novel potato late blight resistance gene from Solanum berthaultii. Theor Appl Genet 112:674-687 
Rauscher G, Simko I, Mayton H, Bonierbale M, Smart CD, Grünwald NJ, Greenland A, Fry WE (2010) Quantitative resistance to late blight from Solanum berthaultii cosegregates with $R_{\text {pi-ber }}$ : insights in stability through isolates and environment. Theor Appl Genet 121:1553-1567

Saski C, Lee SB, Daniell H, Wood TC, Tomkins J, Kim HG, Jansen RK (2005) Complete chloroplast genome sequence of Glycine max and comparative analyses with other legume genomes. Plant Mol Biol 59:309-322

Schwartz S, Kent WJ, Smit A, Zhang Z, Baertsch R, Hardison RC, Haussler D, Miller W (2003) Human-mouse alignments with BLASTZ. Genome Res 13:103-107

Smilde WD, Brigneti G, Jagger L, Perkins S, Jones JDG (2005) Solanum mochiquense chromosome IX carries a novel late blight resistance gene Rpi-mocl. Theor Appl Genet 110: 252-258

Spooner DM, Castillo R(1997) Reexamination of series relationships of South American wild potatoes (Solanaceae: Solanum sect. Petato) : evidence from chloroplast DNA restriction site variation. Am J Botany 84:671-685

Sugiura M, Hirose T, Sugita M (1998) Evolution and mechanism of translation in chloroplast. Annu Rev Genet 32:437-459

Swofford DL (2001) Phylogenetic analysis using parsimony (* and other methods). Sinauer Associates, Sunderland

Symda-Dajmund P, Śliwka J, Wasilewicz-Flis I, Jakuczun H, Zimnoch-Guzowska E (2016) Genetic composition of interspecific potato somatic hybrids and autofused $4 \mathrm{x}$ plants evaluated by DArT and cytoplasmic DNA markers. Plant Cell Rep 35:1345-1358
Tamura K, Stecher G, Peterson D, Filipski A, Kumar S (2013) MEGA6: molecular evolutionary genetic analysis version 6.0. Mol Biol Evol 30:2725-2729

Tan MYA, Hutten RCB, Visser RGF, van Eck HJ (2010) The effect of pyramiding of Phytophthora infestans resistance genes $R_{p i-m c d l}$ and $R_{p i-b e r}$ in potato. Theor Appl Genet 121:117-125

Uncu AT, Celik I, Devran Z, Ozkaynak E, Frary A, Frary A, Doganlar S (2015) Development of a SNP-based CAPS assay for the $\mathrm{Me} 1$ gene conferring resistance to root knot nematode in pepper. Euphytica 206:393-399

Uribe P, Jansky S, Halterman D (2014) Two CAPS markers predict Verticillium wilt resistance in wild Solanum species. Mol Breeding 33:465-476

Wang Y, Liu W, Xu L, Wang Y, Chen Y, Luo X, Tang M, Liu L (2017) Development of SNP markers based on transcriptome sequences and their application in germplasm identification in radish (Raphanus sativus L.). Mol Breeding 37:26

Wyman SK, Jansen RK, Boore JL (2004) Antomatic annotation of organellar genomes with DOGMA. Bioinformatics 20:3252-3255

Xiang F, Xia G, Zhi D, Wang J, Nie H, Chen H(2004) Regeneration of somatic hybrids in relation to the nuclear and cytoplasmic genomes of wheat and Setaria italica. Genome 47:680-688

Yurina NP, Odintsova MS (1998) Comparative structural organization of plant chloroplast and mitochondrial genomes. Russ J Genet 34:5-22

Zeka D, Sedlak P, Sedlakova V, Vasek J, Domkarova J, Vejl P (2014) cpDNA SSR polymorphism in secondary germplasm of potato maintained in Czech gene bank. Pak J Agr Sci 51:743-748 